\title{
ORIGINAL ARTICLE Effects of methylprednisolone and 4-chloro- 3-hydroxyanthranilic acid in experimental spinal cord injury in the guinea pig appear to be mediated by different and potentially complementary mechanisms
}

\author{
JR Yates ${ }^{1,2,3}$, EA Gay ${ }^{1,5}$, MP Heyes ${ }^{4,6}$ and AR Blight ${ }^{1,2}$
}

\begin{abstract}
Study design: Blinded, placebo-controlled, parallel treatment group studies of the effects of methylprednisolone (MP) or 4-chloro3-hydroxyanthranilate (4-Cl-3-HAA) on behavioral outcome and quinolinic acid tissue levels from experimental thoracic spinal cord injury in adult guinea pigs.

Objectives: To compare the effects of treatment with high-dose MP, a corticosteroid, and 4-Cl-3-HAA, a compound that inhibits synthesis of the neurotoxin quinolinic acid (QUIN) by activated macrophages. To explore the effect of different times of treatment using these two approaches to ameliorating secondary tissue damage.
\end{abstract}

Setting: Laboratory animal studies at the University of North Carolina, Chapel Hill, NC, USA.

Methods: Standardized spinal cord injuries were produced in anesthetized guinea pigs, using lateral compression of the spinal cord. Behavioral impairment and recovery were measured by placing and toe-spread responses (motor function), cutaneus trunci muscle reflex receptive field areas and somatosensory-evoked potentials (sensory function). Tissue quinolinic acid levels were measured by gas chromatograph/mass spectrometry.

Results: The current experiments showed a reduction in delayed loss of motor and sensory function in the guinea pig with MP (150 $\mathrm{mg} \mathrm{kg}^{-1}$, intraperitoneally in split doses between 0.5 and $6 \mathrm{~h}$ ), but no significant reduction in tissue QUIN. Improved sensory function was seen with a single dose of $60 \mathrm{mg} \mathrm{kg}^{-1} \mathrm{MP}$ intraperitoneally at $5 \mathrm{~h}$ after injury, but not at $10 \mathrm{~h}$ after injury. A single dose of 4- $\mathrm{Cl}-3-\mathrm{HAA}$ at $5 \mathrm{~h}$ in the guinea pig did not produce the sensory and motor improvements seen in previous studies with 12 days of dosing, beginning at $5 \mathrm{~h}$.

Conclusion: These studies, together with earlier findings, indicate that both drugs can attenuate secondary pathologic damage after $\mathrm{SCl}$, but through separate mechanisms. These are most likely an acute reduction by MP of oxidative processes and reduction by 4-Cl-3-HAA of QUIN synthesis.

Spinal Cord (2014) 52, 662-666; doi:10.1038/sc.2014.118; published online 22 July 2014

\section{INTRODUCTION}

In animal models of spinal cord injury (SCI), long-term functional outcome depends on delayed secondary pathologic mechanisms in addition to direct mechanical damage. Among the proposed contributing mechanisms are decreased blood flow with reperfusion injury, tissue edema, production of reactive oxygen species yielding lipid peroxidation of membranes, and release of other toxic molecules by infiltrating inflammatory cells. ${ }^{1-4}$ Study of secondary mechanisms has been challenging because of the difficulty in obtaining direct evidence of secondary damage other than through the effects of experimental acute interventions on long-term outcome. There has been little direct and unequivocal evidence for secondary tissue damage or neurologic deficits in SCI despite the large amount of research that has been performed to try to prevent it. Earlier studies in a guinea pig model of SCI have been distinct in showing a clear secondary loss of function in the first few days following injury, associated with the inflammatory process. ${ }^{3}$

Corticosteroids have been used in the treatment of central nervous system (CNS) trauma initially with the purpose of reducing edema. Studies in animal models of SCI have shown that the glucocorticoid, methylprednisolone (MP), can prevent lipid peroxidation, ${ }^{1,5}$ help maintain blood flow, ${ }^{6}$ and prevent lactacidosis in CNS tissues, ${ }^{7}$ leading to long-term functional improvement. ${ }^{8}$ Efficacy of MP in these studies depended on delivering doses far in excess of those required for the activation of steroid receptors, and treating within a narrow time window of, at most, a few hours after injury. ${ }^{9}$ In a large multicenter clinical trial, a high dose of MP was reported to improve long-term neurological outcome in a subset of people treated within

${ }^{1}$ Curriculum in Neurobiology and Division of Neurosurgery, University of North Carolina at Chapel Hill, Chapel Hill, NC, USA; ${ }^{2}$ Acorda Therapeutics Inc., Ardsley, NY, USA ${ }^{3}$ Department of Psychology, Ohio Wesleyan University, Delaware, OH, USA and ${ }^{4}$ Laboratory of Neurotoxicology, National Institute of Mental Health, Chapel Hill, NC, USA ${ }^{5}$ Current address: NIEHS, NC, USA.

${ }^{6}$ Current address: Tateioms, Laurel, MD, USA.

Correspondence: Dr JR Yates, Department of Psychology, Ohio Wesleyan University, 61 South Sandusky Street, Delaware, OH 43015, USA

E-mail: jryates@owu.edu

Received 12 January 2014; revised 19 May 2014; accepted 6 June 2014; published online 22 July 2014 
$8 \mathrm{~h}$ of injury. ${ }^{10,11}$ These data have been interpreted as supporting an antioxidant mechanism of action, although MP may also attenuate neurologic deterioration through other mechanisms, including receptor-mediated effects on leukocyte activation.

Studies of SCI in guinea pigs have implicated activated macrophages in secondary tissue damage and delayed loss of function. ${ }^{1,4,12}$ The macrophage toxin, silica, was found to reduce secondary functional loss. ${ }^{2}$ Specifically, production of the neurotoxin quinolinic acid (QUIN), by activated macrophages, is implicated as a factor in the evolution of these secondary functional deficits. ${ }^{3}$ Inhibition of QUIN synthesis with systemic administration of high doses of 4-chloro-3-hydroxyanthranilic acid (4-Cl-3-HAA) reduced secondary loss of function. ${ }^{13}$ Delivery of $4-\mathrm{Cl}-3-\mathrm{HAA}$ as late as $5 \mathrm{~h}$ after injury reduced secondary functional deficits while approximately doubling the survival of white matter at the injury site. ${ }^{3}$ However, 4-Cl-3-HAA may also have some antioxidant activity. Similarly, MP has anti-inflammatory activity separate from its antioxidant effects, complicating the identification of the mechanisms of action for these two.

The purpose of this study was to determine whether MP influences the secondary functional decline after primary injury in the guinea pig model, and to explore the time window of treatment for any beneficial effect. We also measured parenchymal QUIN concentration, both to use QUIN concentrations as a biochemical marker of macrophage/microglial activation and to evaluate whether effects of methyprednisolone on neurologic status might be mediated through modulation of QUIN and/or macrophage activity.

\section{MATERIALS AND METHODS}

\section{Animals and surgeries}

Female Hartley guinea pigs $(460 \pm 105 \mathrm{~g})$ were used in three experiments (Table 1). Subjects were housed two per cage and had access to food and water ad libitum. In Experiment 1, half of the animals underwent two surgeries, one to implant electrodes for somatosensory-evoked potentials (SEP) data collection and a second for SCI. In the remainder of Experiment 1 and in Experiments 2 and 3, animals underwent surgery for SCI. The methods were approved by the Institutional Animal Care and Use Committee of the University of North Carolina at Chapel Hill.

Animals were anesthetized with $60 \mathrm{mg} \mathrm{kg}^{-1}$ ketamine $\mathrm{HCl}, 10 \mathrm{mg} \mathrm{kg}^{-1}$ xylazine and $0.6 \mathrm{mg} \mathrm{kg}^{-1}$ acepromazine maleate. In Experiment 1, the skull over the sensory cortex was exposed and electrodes for recording SEPs were implanted. Gold-plated, round-ended, $0.8 \mathrm{~mm}$ diameter pins were inserted bilaterally to just above the somatosensory cortex, $4-5 \mathrm{~mm}$ lateral and $1-2 \mathrm{~mm}$ caudal of bregma. Three additional holes were drilled $10 \mathrm{~mm}$ rostral, $1-2 \mathrm{~mm}$ right lateral and $5 \mathrm{~mm}$ caudal and $1-2 \mathrm{~mm}$ left lateral for stainless-steel screws ( $1 \mathrm{~mm}$ in diameter and $5 \mathrm{~mm}$ in length). A dental acrylic cap was applied, with the top of the pins and the rostral screw exposed for connection to the recording amplifier and the skin was closed around the cap. After 2-3 days, injury surgery was performed as described in full in previous publications. ${ }^{2-4,12}$ Briefly, a lateral compression injury of thoracic segment 12 was performed using modified forceps. The cord was compressed to a thickness of $1.2 \mathrm{~mm}$ over $5 \mathrm{~mm}$ of its length for $15 \mathrm{~s}$. Animals in Experiment 1, used for SEP recording, were anesthetized with one-half the normal surgical dose and preinjury SEPs were recorded. Animals were then given an additional twothirds of the normal surgical dose of anesthesia for injury surgery. For the remainder of Experiment 1 and for Experiments 2 and 3, animals were given a single surgical dose of anesthesia before surgery.

\section{Drug treatment}

In Experiment 1, one treatment group received $60 \mathrm{mg} \mathrm{kg}^{-1} \mathrm{MP}$ or vehicle intraperitoneally at $30 \mathrm{~min}$ after injury and $30 \mathrm{mg} \mathrm{kg}^{-1} \mathrm{MP}$ at 2,4 and $6 \mathrm{~h}$ after injury ( $n=13$ for MP and vehicle) $(60 / 30 / 30 / 30) .{ }^{14}$ This dosing was based on the work of Behrmann et al., ${ }^{14}$ which found an intravenous regimen of 60/30/ 30/30 mg MP to be superior to other doses and treatments in terms of open field walking and incline plane evaluations in rats. Blood levels achieved with the intraperitoneal administration used in the present study are likely to have been somewhat lower and slower to peak than those reached with intravenous administration. The second group received $30 \mathrm{mg} \mathrm{kg}^{-1} \mathrm{MP}$ or vehicle at $30 \mathrm{~min}$ after injury and $15 \mathrm{mg} \mathrm{kg}^{-1}$ at 2, 4 and 6 hours after injury $(n=7$ for MP and vehicle) (30/15/15/15), again similar to one of the intravenous regimens used by Behrmann et al. ${ }^{14}$ The 60/30/30/30 dosing regimen was used for Experiment 2 ( $n=5$ for MP and vehicle) to determine the effect of MP on 3-day QUIN accumulation. In Experiment 3, designed to explore further the time window of efficacy of MP, one group of animals received one injection of MP $\left(60 \mathrm{mg} \mathrm{kg}^{-1}\right)$ or vehicle solution at $5 \mathrm{~h}$ after injury. A second group received either a single injection of $33 \mathrm{mg} 4$-Cl-3-HAA or an equivalent volume of vehicle at $5 \mathrm{~h}$ after injury. A third group received either MP $(60 \mathrm{mg} \mathrm{kg})$ or vehicle at $10 \mathrm{~h}$ after injury.

The 4-chloro-3-hydroxyanthranilate (4-Cl-3-HAA) was synthesized at the Laboratory of Neurotoxicology at NIMH from commercially available 2-chloro-5-methylyphenol. After methylation of the hydroxyl group, the benzylic methyl group was oxidized, followed by nitration and chromatographic separation of the unwanted isomer. The nitro group was then reduced, followed by protection of the hydroxyl group to produce 4-Cl-3-HAA. Immediately before injection, $26 \mathrm{mg}$ of $4-\mathrm{Cl}-3-\mathrm{HAA}$ was dissolved in $2 \mathrm{ml}$ of $0.15 \mathrm{M}$ sodium hydroxide. To this mixture was added $0.1 \mathrm{ml}$ of $0.15 \mathrm{M}$ sodium phosphate (NaH2PO4) in water, followed by $\sim 0.7 \mathrm{ml}$ of $0.1 \mathrm{M} \mathrm{HCl}$ to a final $\mathrm{pH}$ of 7.4. The vehicle solution contained all components except the 4-Cl-3-HAA (pH 7.4).

Table 1 Number of animals used in each experiment in the study and which experimental outcomes were measured

\begin{tabular}{|c|c|c|c|c|c|}
\hline Experiment & Drug treatment & Behavior & SEPS & QUIN & KYNA \\
\hline \multirow[t]{4}{*}{ 1. Effect of MP on QUIN and function } & MP 60/30/30/30 & 13 & 13 & 6 & 6 \\
\hline & Vehicle 60/30/30/30 & 13 & 13 & 6 & 6 \\
\hline & MP 30/15/15/15 & 7 & 7 & 5 & 5 \\
\hline & Vehicle 30/15/15/15 & 7 & 7 & 5 & 5 \\
\hline \multirow[t]{2}{*}{ 2. Effect of MP on QUIN at 3 days after injury } & MP 60/30/30/30 & 5 & & & \\
\hline & Vehicle 60/30/30/30 & 5 & & & \\
\hline \multirow[t]{6}{*}{ 3. Effect of single injection of MP or 4-Cl-3-HAA on behavior } & MP $605 \mathrm{~h}$ & 5 & & & \\
\hline & Vehicle $605 \mathrm{~h}$ & 5 & & & \\
\hline & 4-Cl-3-HAA $5 \mathrm{~h}$ & 6 & & & \\
\hline & Buffer $5 \mathrm{~h}$ & 6 & & & \\
\hline & MP $6010 \mathrm{~h}$ & 6 & & & \\
\hline & Vehicle $10 \mathrm{~h}$ & 6 & & & \\
\hline
\end{tabular}

Abbreviations: 4-Cl-3-HAA, 4-chloro-3-hydroxyanthranilate; KYNA, kynurenic acid; MP, methylprednisolone; QUIN, neurotoxin quinolinic acid; SEP, somatosensory-evoked potentials. 


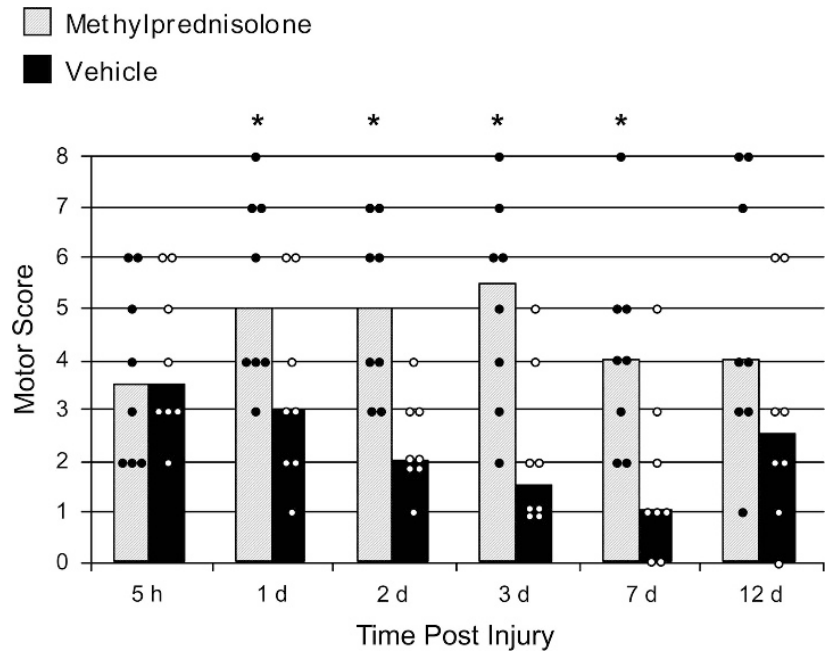

Figure 1 Histogram illustrating the individual (dots) and median (bars) motor scores for high-dose (60/30/30/30 $\mathrm{mg} \mathrm{kg}^{-1}$ ) MP-treated guinea pigs and vehicle-treated guinea pigs over time after injury. Motor scores were significantly different between treatments at $1,2,3$, and 7 days after injury, as analyzed by the Mann-Whitney $U$-test $\left({ }^{*} P<0.05\right)$.

\section{Behavioral and electrophysiologic monitoring}

Recovery was monitored using sensory and motor functional tests and, in some animals, SEPs. ${ }^{12}$ Hind-limb toe-spread responses and proprioceptive placing were used to examine motor function. ${ }^{3,4,12}$ Sensory function was tested using the cutaneus trunci muscle (CTM) reflex receptive field area. ${ }^{3,4}$

Toe-spread and proprioceptive placing responses. These functional assessments were performed as described previously. ${ }^{3,4}$ Briefly, holding the animal elevated and moving it briskly downward or rapidly turning the animal over while suspended elicited the toe-spread response. Toe-spread responses in each hind limb were graded as normal, weak, or not detectable and scored on each leg as 2,1 , or 0 , respectively. The reflexive proprioceptive placing response to a gentle extension of each hind limb was also examined. Scoring of placing responses was as for the toe-spread response: normal, weak, or absent.

CTM. The area of the receptive field of the CTM reflex was measured. ${ }^{3,4}$ The receptive field was marked on the skin for digitization and area measurement.

SEPs. SEPs were recorded preinjury, and $1 \mathrm{~h}, 1$ day and 12 days after injury. Animals were anesthetized with one-half of the surgical dose of anesthesia. Recordings were taken from the pins over the somatosensory cortex and referenced to the rostral screw. The animals were grounded using a disc electrode placed on the shaved neck with electrode gel. Animals were stimulated using $5 \mathrm{~mm}$ surface electrodes placed over the calcaneus bone (anode) and half way up the tibia (cathode). Both hind limbs were stimulated simultaneously. A square-wave stimulus pulse of $0.1 \mathrm{~ms}$ was applied and the response averaged over 128 stimuli. Three averages were recorded using $7 \mathrm{~mA}$ stimulating pulse and then another three averages with a $10 \mathrm{~mA}$ stimulating pulse. Response peaked at $7 \mathrm{~mA}$, and $10 \mathrm{~mA}$ trials were used to ensure that maximal response amplitudes were obtained. The key parameters of the waveforms for each side of the cortex were averaged. The largest amplitude responses from the two stimulus intensities and corresponding latencies were used for each time point.

Biochemical analysis. Samples of CSF, serum, CNS, and systemic tissues were collected 12 days after injury. Samples of liver, lung, spleen, and kidney were also collected. Tissues were collected and QUIN was quantified as previously described. ${ }^{5,15,16}$

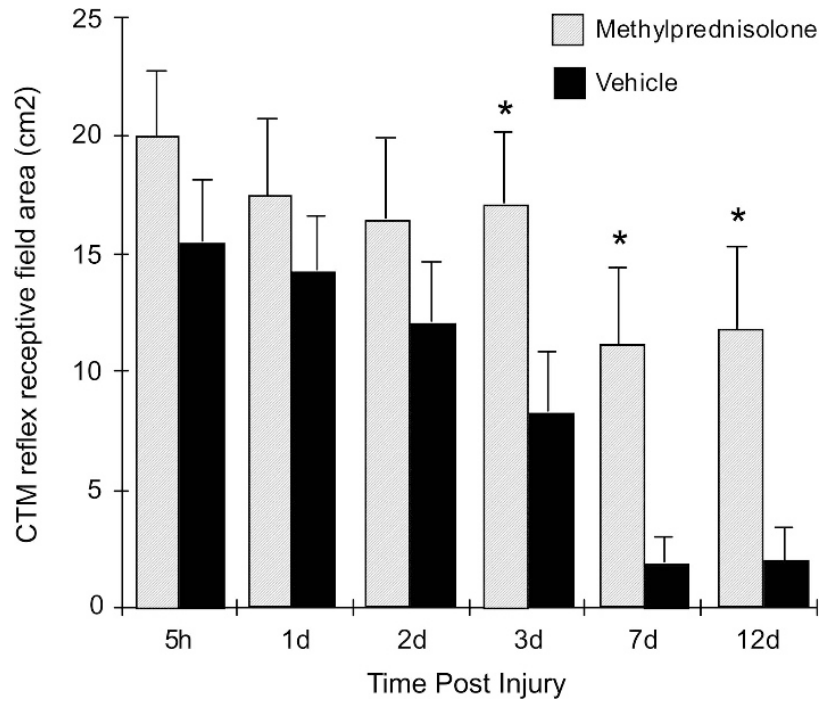

Figure 2 Histogram to show the mean ( \pm s.e.m.) areas for the CTM reflex for high-dose MP-treated animals and vehicle-treated animals over time after injury. MP-treated animals maintained significantly larger CTM reflex receptive fields than vehicle-treated animals at 3,7 , and 12 days after injury ( ${ }^{*} P<0.05$, ANOVA and Bonferroni-Dunn post hoc test).

\section{RESULTS}

\section{Experiment 1}

Motor function. Vehicle-treated animals exhibited a pattern of immediate functional decline, reflecting the initial mechanical damage at the injury site, followed by delayed, secondary decline in motor function (Figure 1). Animals treated on the 60/30/30/30 MP dosing schedule showed statistically significant $(P<0.05$; analysis of variance (ANOVA) and Bonferroni-Dunn post hoc analysis) attenuation of this secondary decline in function at $1,2,3$, and 7 days after injury (Figure 1). The 30/15/15/15 MP dosing regimen in Experiment 1 did not significantly affect secondary loss of motor function (data not shown).

Sensory function. The 60/30/30/30 MP treatment protocol was also associated with a significantly decreased secondary decline in CTM reflex receptive field area compared with vehicle-treated animals (Figure 2). This effect was significant at 3, 7, and 12 days after injury $(P<0.05$; ANOVA and Bonferroni-Dunn post hoc analysis). Again, the secondary decline in ascending sensory function was not significantly attenuated by the lower dose, although there were fewer animals and unequal sensory deficits were seen after injury.

Electrophysiologic function. Neither treatment protocol resulted in a significant difference from preinjury amplitude of SEPs at the postinjury time points (data not shown). There was a trend toward increased amplitude of the SEP in the 60/30/30/30 MP-treated group compared with the vehicle-treated animals at both 1 day $(P=0.08$; ANOVA and Bonferroni-Dunn post hoc analysis) and 12 days after injury ( $P=0.06$; ANOVA and Bonferroni-Dunn post hoc analysis).

Effects of MP on parenchymal QUIN responses to injury. At 12 days after injury, parenchymal QUIN concentrations were increased, with peak values located in the area of injury (Figure 3). Methyprednisolone had no effect on the accumulation of QUIN concentrations at 12 days after injury or on its regional profile along the cord. 


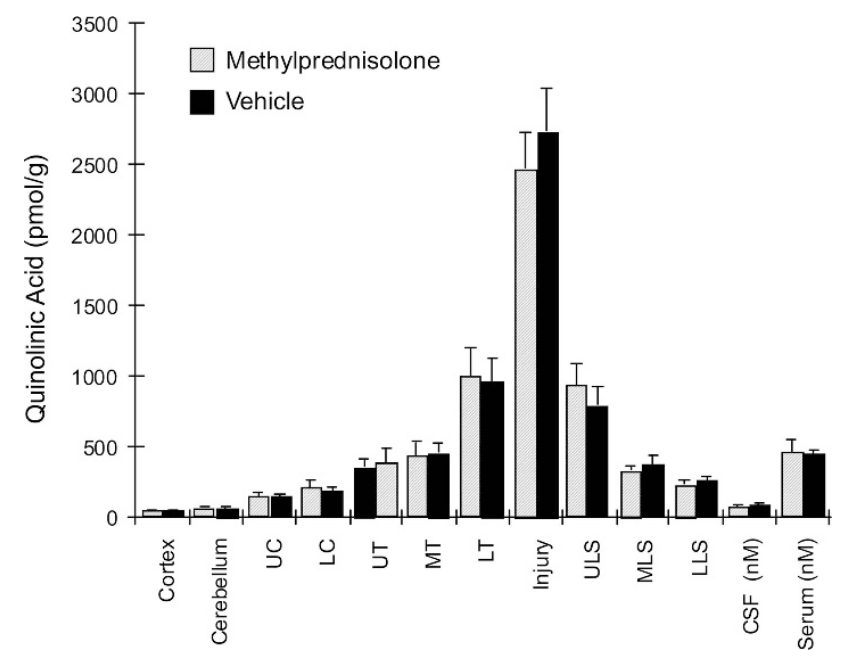

Figure 3 Histogram illustrating tissue and fluid QUIN concentrations at 12 days after injury for the high-dose MP and associated vehicle groups. Tissue samples were obtained from the full extent of the central nervous system (cerebral cortex, cerebellum, upper and lower cervical (UC, LC), upper, mid and lower thoracic, and lumbosacral (UT, MT, LT, ULS, MLS, LLS) spinal cord, the injury site itself, and cerebrospinal fluid and serum. No significant differences were found between the two groups in any of these measures, using ANOVA. Similar results were obtained in the lower dose MP group versus vehicle (not shown).

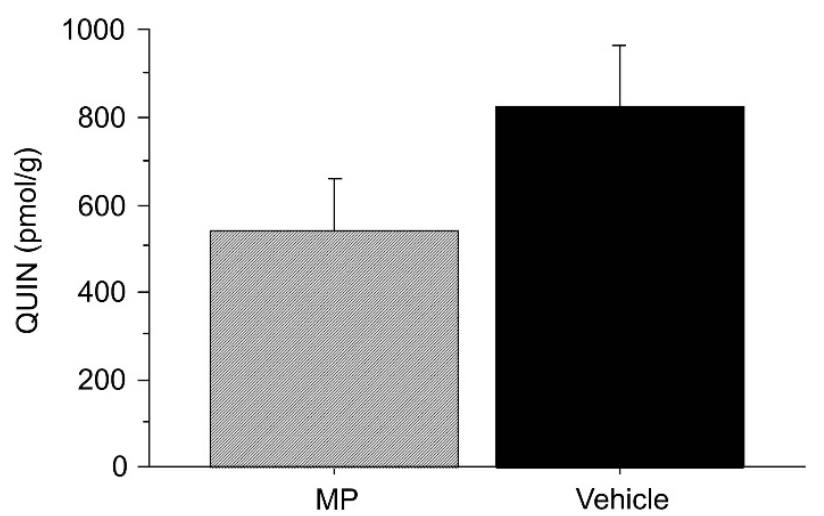

Figure 4 Histogram illustrating injury site QUIN concentrations at 3 days after injury for the high-dose MP and vehicle groups. No significant differences were found between the two groups.

\section{Experiment 2}

To determine whether MP might affect QUIN levels earlier after injury than 12 days, the effect of MP at 3 days was investigated. No significant difference between QUIN levels in the two treatment groups was seen at this time $(P=0.16)$, but the average QUIN accumulation at the injury site was higher in the vehicle-treated group, with $543.6 \pm 119.0$ and $827.7 \pm 140.0 \mathrm{pmolg}^{-1}$ (mean \pm s.e.m.) in MP and vehicle groups, respectively (Figure 4).

\section{Experiment 3}

A single injection of MP given at $5 \mathrm{~h}$ after injury, even in a small group of animals $(n=5)$, showed statistically significant improvement compared with vehicle treatment $(n=5)$ in the CTM reflex receptive field at 3 days after injury (Figure 5). There appeared to be attenuation and delay of secondary decline at all time points, and the lack of statistical significance was probably the result of the small

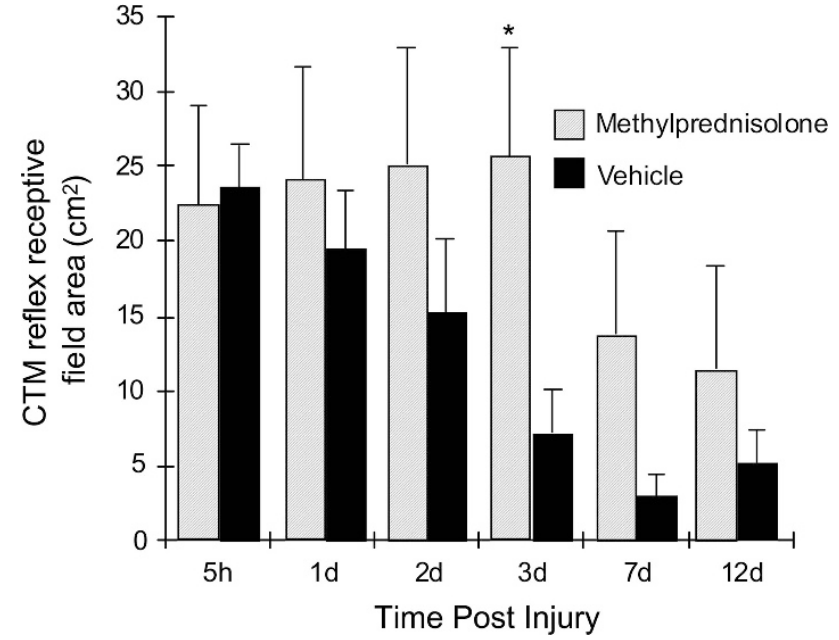

Figure 5 Histogram illustrating the effect of single injection of $60 \mathrm{mg} \mathrm{kg}^{-1}$ $\mathrm{MP}$ at $5 \mathrm{~h}$ after injury on secondary loss of CTM reflex receptive field area in guinea pigs. There appeared to be a delay and attenuation of loss of the sensory field in the MP-treated animals compared with the vehicle-treated group, although the differences were only significant at 3 days after injury, possibly as a result of small group sizes.

sample size in this experiment. No beneficial effect was seen with a single injection of MP at $10 \mathrm{~h}$ after injury ( $n=6$ per treatment group, data not shown). Attenuation of secondary decline was not seen with a single injection of 4-Cl-3-HAA at $5 \mathrm{~h}$ compared with buffer vehicle treatment ( $n=6$ per group, data not shown).

\section{DISCUSSION}

These studies support the conclusion that two distinct mechanisms of secondary pathology are addressed by treatment with 4-Cl-3-HAA and MP. Although high-dose MP attenuates behavioral deficits in a manner similar to that previously shown with 4-Cl-3-HAA in the guinea pig, there was no significant attenuation of QUIN accumulation.

The guinea pig model was used to evaluate the time course and magnitude of the effects of MP on secondary functional deficits after SCI, because secondary neurologic deficits after SCI can be observed and quantified in this species. ${ }^{3}$ Previous studies in other species have established that MP treatment within the first few hours after injury reduces the magnitude of the prompt decrease in blood flow, ${ }^{7}$ prevents the degradation of neurofilaments ${ }^{17}$ and acute tissue damage, ${ }^{18}$ reduces disturbances in ion distribution, ${ }^{1}$ attenuates the invasion of neutrophils and macrophages, ${ }^{19}$ and inhibits lipid peroxidation. ${ }^{5}$ These observations led to the hypothesis that the beneficial effects of MP on neurologic function result from these immediate physiologic and biochemical changes. The present results demonstrate, in a species that has behavioral correlates to secondary pathology, that the beneficial effects of MP on neurologic function do not result in an immediate improvement in functional status. This was evident for motor function at $5 \mathrm{~h}$ after injury, $4.5 \mathrm{~h}$ after the first treatment (Figure 1) and at $5 \mathrm{~h}, 1$ day and 2 days for sensory function (Figure 2). Therefore, the neurologic improvements appear to be delayed until long after the observed improvements in blood flow, ion distribution, and lipid peroxidation. Given the short plasma half-life of MP, these observations seem most compatible with the hypothesis that the improvements are indirectly linked, perhaps to earlier changes in blood flow and metabolism, in ways that require time for their full expression.

In guinea pigs and humans, macrophages that infiltrate injured tissue and activated microglia have a high capacity to synthesize 
QUIN, ${ }^{13,20-22}$ whereas astrocytes and neurons have a much lower capacity for QUIN synthesis. ${ }^{22,23}$ In addition, while CNS diseases involving immune activation are associated with elevations in QUIN concentration secondary to increased local production, diseases primarily associated with astrogliosis are not. ${ }^{21}$ Therefore, tissue concentrations of QUIN can be considered as a surrogate marker of parenchymal macrophage invasion and/or microglial activation. MP had no significant effect on parenchymal QUIN concentration in response to SCI 12 days after injury, indicating that at this time point, the acute treatment with MP did not significantly affect either the degree of macrophage invasion or microglial activation. The slight and nonsignificant reduction in QUIN concentrations at 3 days (Figure 4) and 12 days (Figure 3) after injury may be consistent with a reduction of phagocytes seen in some studies in rat models. ${ }^{19}$ Any reduction or delay in the inflammatory process, however, does not result in persisting attenuation of QUIN levels. These results appear to contrast with Saito and co-workers ${ }^{23}$ who showed dexamethasone treatment after cerebral ischemia in the gerbil resulted in significant suppression of the QUIN accumulation at 7 days after injury. These results do however support results in another model of CNS inflammation in the guinea pig (experimental autoimmune encephalomyelitis) where Karlik et al. ${ }^{24}$ demonstrated that MP administration had no effect on CNS inflammation. If this interpretation is correct, namely that MP had minimal effect on macrophage/microglial activation during the course of secondary neurologic decline in function, then the window of opportunity and the target for therapeutic intervention in SCI via this cell-mediated mechanism may remain open to other interventions.

There was a beneficial effect of a single injection of MP at $5 \mathrm{~h}$ after injury, but not at $10 \mathrm{~h}$. These preliminary findings are consistent with the 8-h treatment window for MP derived from clinical studies. ${ }^{10,11}$ This kind of match between animal model and clinical data is reassuring for the ability to translate findings from the laboratory to the clinic. The lack of effect of a single injection of 4-Cl-3-HAA at $5 \mathrm{~h}$ after injury supports the hypothesis that the beneficial effects of continued 4-Cl-3-HAA treatment in the guinea pig demonstrated in earlier studies ${ }^{5,12}$ are mediated by blockade of QUIN rather than any early antioxidant effect. It is therefore likely that 4-Cl-3-HAA has a longer therapeutic window than MP, because the peak of QUIN accumulation does not occur until 12 days after injury. ${ }^{25}$ These indications should ${ }^{26-28}$ be considered when and if therapeutic interventions targeting QUIN accumulation are tested clinically.

Taken together, these studies indicate that MP and 4-Cl-3-HAA act via different mechanisms to attenuate secondary behavioral deficits and tissue destruction in the guinea pig model. These mechanisms result in similar behavioral benefits and it remains to be determined if the two approaches can be used together and whether they may act synergistically.

\section{DATA ARCHIVING}

There were no data to deposit.

\section{CONFLICT OF INTEREST}

The authors declare no conflict of interest.

\section{ACKNOWLEDGEMENTS}

This work was supported in part by Grant NS-33687 from NIH, NINDS, by Grant 1793 from the Spinal Cord Research Foundation of the Paralyzed Veterans of America and by research contract BA2-9701 from the American Paralysis Association.
1 Hausmann O. Post-traumatic inflammation following spinal cord injury. Spinal Cord 2003; 41: 369-378.

2 Blight AR, Saito K, Heyes MP. Increased levels of the excitotoxin quinolinic acid in spinal cord following contusion injury. Brain Res 1993; 632: 314-316.

3 Blight AR. Effects of silica on the outcome from experimental spinal cord injury: implication of macrophages in secondary tissue damage. Neuroscience 1994; 60: 263-273.

4 Yates JR, Heyes MP, Blight AR. 4-Chloro-3-hydroxyanthranilate reduces loca quinolinic acid synthesis, improves functional recovery and preserves white matter after spinal cord injury. J Neurotrauma 2006; 23: 866-881.

5 Hall ED. Antioxidant therapies for acute spinal cord injury. Neurotherapeutics 2001; 8 152-167.

6 Young W, Flamm ES. Effect of high-dose corticosteroid therapy on blood flow, evoked potentials, and extracellular calcium in experimental spinal injury. J Neurosurg 1982; 57: 667-673

7 Braughler JM, Hall ED. Lactate and pyruvate metabolism in injured cat spinal cord before and after a single large intravenous dose of methylprednisolone. J. Neurosurg 1983; 59: 256-261.

8 Hall ED, Springer JE. Neuroprotection and acute spinal cord injury: a reappraisal. NeuroRx 2004; 1: 80-100.

9 Braughler JM, Hall ED, Means ED, Waters TR, Anderson DK. Evaluation of an intensive methylprednisolone sodium succinate dosing regimen in experimental spinal cord injury. J Neurosurg 1987; 67: 102-105.

10 Bracken MB, Shepard MJ, Collins WF, Holford TR, Young W, Baskin DS et al. A randomized, controlled trial of methylprednisolone or naloxone in the treatment of acute spinal-cord injury. New Engl J Med 1990; 322: 1405-1411.

11 Bracken MB, Shepard MJ, Holford TR, Leo-Summers L, Aldrich EF, Fazl M et al. Administration of methylprednisolone for 24 or $48 \mathrm{~h}$ or tirilazad mesylate for $48 \mathrm{~h}$ in the treatment of acute spinal cord injury: Results of the third National Acute Spinal Cord Injury randomized controlled trial. JAMA 1997; 277 1597-1604.

12 Blight AR, Cohen TI, Saito K, Heyes MP. Quinolinic acid accumulation and functional deficits following experimental spinal cord injury. Brain 1995; 118: 735-752.

13 Ozaki Y, Edelstein MP, Duch DS. The actions of interferon and antiinflammatory agents of induction of indoleamine 2,3-dioxygenase in human peripheral blood monocytes. Biochem Biophys Res Commun 1987; 144: 1147-1153.

14 Behrmann DL, Bresnahan JC, Beattie MS. Modeling of acute spinal cord injury in the rat: neuroprotection and enhanced recovery with methylprednisolone, U-74006F and YM-14673. Exp Neurol 1994; 126: 61-75.

15 Heyes MP, Quearry BJ. Quantification of kynurenic acid in cerebrospinal fluid: effects of systemic and central L-kynurenine administration. J Chromatogr 1990; 530: 108-115.

16 Heyes MP, Markey SP. Quantification of quinolinic acid in the rat brain, whole blood and plasma by gas chromatography and negative chemical ionization mass spectrometry: effects of systemic L-tryptophan administration on brain and blood quinolinic acid concentrations. Anal Biochem 1988; 174: 349-359.

17 Braughler JM, Hall ED. Effects of multi-dose methylprednisolone sodium succinate administration on injured cat spinal cord neurofilament degradation and energy metabolism. J Neurosurg 1984; 61: 290-295.

18 Constantini S, Young W. The effects of methylprednisolone and the ganglioside GM1 on acute spinal cord injury in rats. J Neurosurg 1994; 80: 97-111.

19 Bartholdi D, Schwab ME. Methylprednisolone inhibits early inflammatory processes but not ischemic cell death after experimental spinal cord lesion in the rat. Brain Res 1995; 672: 177-186.

20 Werner ER, Bitterlich G, Fuchs D, Hausen A, Reibnegger G, Szabo G et al. Human macrophages degrade tryptophan upon induction by interferon-gamma. Life Sci 1987 41: 273-280.

21 Heyes MP, Saito K, Markey SP. Human macrophages convert L-tryptophan to the neurotoxin quinolinic acid. Biochem J 1992; 283: 633-635.

22 Heyes MP, Chen CY, Major EO, Saito K. Different kynurenine pathway enzymes limit quinolinic acid formation by various human cell types. Biochem J 1997; 326: 351-356.

23 Heyes MP, Saito K, Chen CY, Proescholdt MG, Nowak TS Jr, Li J et al. Species heterogeneity between gerbils and rats: quinolinate production by microglia and astrocytes and accumulations in response to ischemic brain injury and systemic immune activation. J Neurochem 1997; 69: 1519-1529.

24 Karlik SJ, Stavraky RT, Hall ED. Comparison of tirilazad mesylate (U-74006F) and methylprednisolone sodium succinate treatments in experimental allergic encephalomyelitis in the guinea pig. Mult Scler 1996; 1: 228-235.

25 Blight AR, Leroy EC, Heyes MP. Quinolinic acid accumulation in injured spinal cord: time course, distribution and species differences between rat and guinea pig J Neurotrauma 1997; 14: 89-98.

26 Blight AR, McGinnis ME, Borgens RB. Cutaneus trunci muscle reflex of the guinea pig. J Comp Neurol 1990; 296: 614-633.

27 Heyes MP, Achim CL, Wiley CA, Major EO, Saito K, Markey SP. Human microglia convert L-tryptophan into the neurotoxin quinolinic acid. Biochem J 1996; 320: 595-597.

28 Saito K, Seishima M, Noma A, Suyama K, Markey SP, Heyes MP. 4-Chloro-3-hyrdoxyanthranilate attenuates quinolinic acid accumulation in brain following transient cerebral ischemia in the gerbil. Adv Exp Med Biol 1996; 398: 407-411. 